\title{
A Comparative Study on the Interaction of Sulfonamide and Nanosulfonamide with Human Serum Albumin
}

\author{
G. Rezaei Behbehani, ${ }^{1}$ Moayed Hossaini Sadr, ${ }^{2}$ H. Nabipur, ${ }^{2}$ and L. Barzegar ${ }^{3}$ \\ ${ }^{1}$ Chemistry Department, Imam Khomeini International University, Qazvin, Iran \\ ${ }^{2}$ Chemistry Department, Faculty of Science, Azarbaijan Shahid Madani University, Tabriz, Iran \\ ${ }^{3}$ Chemistry Department, Faculty of Science, Islamic Azad University, Takestan Branch, Takestan, Iran \\ Correspondence should be addressed to G. Rezaei Behbehani; grb402003@yahoo.com
}

Received 22 June 2012; Revised 27 November 2012; Accepted 11 December 2012

Academic Editor: Yoshihiro Kudo

Copyright (C) 2013 G. Rezaei Behbehani et al. This is an open access article distributed under the Creative Commons Attribution License, which permits unrestricted use, distribution, and reproduction in any medium, provided the original work is properly cited.

\begin{abstract}
Binding parameters of the N-phenyl benzene sulfonyl hydrazide, sulfonamide, and nanosulfonamide interaction with human serum albumin were determined by calorimetry method. The obtained binding parameters indicated that sulfonamide in the second binding sites has higher affinity for binding than the first binding sites. The binding process of sulfonamide to HSA is both enthalpy and entropy driven. The associated equilibrium constants confirm that sulfonamide binds to HSA with high affinity $\left(2.2 \times 10^{6}\right.$ and $3.8610^{5} \mathrm{M}^{-1}$ for first and second sets of binding sites, resp.). The obtained results indicate that sulfonamide increases the HSA antioxidant property. Nanosulfonamide has much more affinity for HSA $\left(3.6 \times 10^{6} \mathrm{M}^{-1}\right)$ than sulfonamide.
\end{abstract}

\section{Introduction}

Physicochemical properties of nanoparticles such as their small size, large surface area, surface charge, and ability to make them potential delivery systems for effective treatments. The pharmacokinetic parameters of therapeutic drugs against the diseases show limitations in their efficacy. The poor bioavailability, side effects due to the high doses administered, long treatment, and the emergence of drug resistant strains are the disadvantages of ordinary drugs. The advances that nanotechnology-based drug delivery systems have made in improving the pharmacokinetics and efficacy of therapeutic drugs [1-4].

Sulfonamides were the first chemical substances systematically used to treat and prevent bacterial infections in humans. Sulfonamides are bacteriostatic drugs; they work by inhibiting the growth and multiplication of bacteria without killing them. Currently, their most common use in humans is treating urinary tract infections [5]. They are estimated to be $16-21 \%$ of annual antibiotic usage, making them the most important group of antibiotics consumed by humans [6]. Sulfonamides are compounds that contain sulfur in a
$\mathrm{SO}_{2} \mathrm{NH}_{2}$ moiety directly attached to a benzene ring. The term "sulfa allergy" is often incorrectly applied to all adverse reactions that occur with sulfonamide-containing medications and not just to those due to hypersensitivity mechanisms. Patients who experience side effects such as nausea and vomiting may interpret this as an allergy and subsequently report that they are allergic to sulfas [7]. The binding of the sulfonamides to serum albumins, an important factor of the pharmacokinetic of these drugs, has been extensively studied by several workers, especially regarding the extent of binding, the stoichiometry, and the influence of the chemical structure on the binding. But only little information is available on the mechanism of the binding and on the nature of the sulfonamide-albumin complex. Some workers have shown a correlation between the partition coefficients of the sulfonamides and the extent of the binding and concluded that the binding is mainly hydrophobic [8]. In this work, we compared the most comprehensive study on the interactions of sulfonamide and nanosulfonamide ( $\mathrm{N}$-phenyl benzene sulfonyl hydrazide) with HSA for further understanding of 
their effects on the stability and the structural changes of the HSA molecules.

\section{Materials and Method}

Human serum albumin (HSA; MW $=66411 \mathrm{gr} / \mathrm{mol}$ ) and Tris buffer used were of analytical grade with the highest purity available without any purification. Sulfonamide derivative (N-phenyl benzene sulfonyl hydrazide) was synthesized. The isothermal titration microcalorimetric experiments were performed with the four-channel commercial microcalorimetric system. Sulfonamide and nanosulfonamide solutions $(1612.9 \mu \mathrm{M})$ were injected by the use of a Hamilton syringe into the calorimetric titration vessel, which contained $1.8 \mathrm{~mL}$ HSA $(60.22 \mu \mathrm{M})$. Injection of sulfonamide solution into the perfusion vessel was repeated 29 times, with $10 \mu \mathrm{L}$ per injection. The calorimetric signal was measured by a digital voltmeter that was part of a computerized recording system. The heats of each injection was calculated by the “Thermometric Digitam 3" software program. The heat of dilution of the sulfonamide and nanosulfonamide solutions were measured as described above except HSA was excluded. The microcalorimeter was frequently calibrated electrically during the course of the study.

\section{Results and Discussion}

We have shown previously that the heats of the ligand + HSA interactions in the aqueous solvent mixtures, can be calculated via the following equation [9-14]:

$$
\begin{aligned}
q= & q_{\max } x_{B}^{\prime}-\delta_{A}^{\theta}\left(x_{A}^{\prime} L_{A}+x_{B}^{\prime} L_{B}\right) \\
& -\left(\delta_{B}^{\theta}-\delta_{A}^{\theta}\right)\left(x_{A}^{\prime} L_{A}+x_{B}^{\prime} L_{B}\right) x_{B}^{\prime},
\end{aligned}
$$

where $q$ are the heats of sulfonamide + HSA or nanosulfonamide + HSA interactions, and $q_{\max }$ represents the heat value upon saturation of all HSA. The parameters $\delta_{A}^{\theta}$ and $\delta_{B}^{\theta}$ are the indexes of HSA stability in the low and high sulfonamide concentrations, respectively. Cooperative binding requires that the macromolecule has more than one binding site, since cooperativity results from the interactions between identical binding sites with the same ligand. If the binding of a ligand at one site increases the affinity for that ligand at another site, then the macromolecule exhibits positive cooperativity. Conversely, if the binding of a ligand at one site lowers the affinity for that ligand at another site, then the enzyme exhibits negative cooperativity. If the ligand binds at each site independently, then the binding is noncooperative. $p>1$ or $p<1$ indicate positive or negative cooperativity of a macromolecule for binding with a ligand, respectively; $p=$ 1 indicates that the binding is noncooperative. $x_{B}^{\prime}$ can be expressed as follows:

$$
x_{B}^{\prime}=\frac{p x_{B}}{x_{A}+p x_{B}}
$$

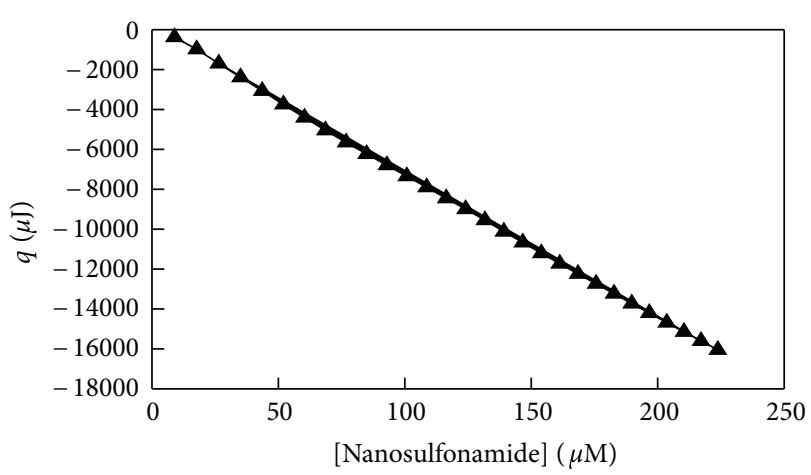

Figure 1: Comparison between the experimental heats ( $\mathbf{\Delta})$ at $300 \mathrm{~K}$, for (nanosulfonamide + HSA) interactions and the calculated data (lines) via (1).

where $x_{B}^{\prime}$ is the fraction of bound sulfonamide or nanosulfonamide to HSA, and $x_{A}^{\prime}=1-x_{B}^{\prime}$ is the fraction of unbound sulfonamide or nanosulfonamide. We can express $x_{B}$ fractions, as the sulfonamide concentrations divided by the maximum concentration of the sulfonamide or nanosulfonamide upon saturation of all HSA as follows:

$$
x_{B}=\frac{[\text { sulfonamide }]}{[\text { sulfonamide }]_{\max }}, \quad x_{A}=1-x_{B},
$$

where [sulfonamide] is the concentration of sulfonamide after every injection, and [sulfonamide $]_{\max }$ is the maximum concentration of the sulfonamide upon saturation of all HSA. $L_{A}$ and $L_{B}$ are the relative contributions of unbound and bound sulfonamide in the heats of dilution in the absence of HSA and can be calculated from the heats of dilution of sulfonamide or nanosulfonamide in buffer, $q_{\text {dilut }}$, as follows:

$$
L_{A}=q_{\text {dilut }}+x_{B}\left(\frac{\partial q_{\text {dilut }}}{\partial x_{B}}\right), \quad L_{B}=q_{\text {dilut }}-x_{A}\left(\frac{\partial q_{\text {dilut }}}{\partial x_{B}}\right)
$$

The heats of sulfonamide + HSA interactions, $q$, were fitted to (1) across the entire sulfonamide or nanosulfonamide compositions. In the fitting procedure, $p$ was changed until the best agreement between the experimental and calculated data was approached (Figures 1 and 2). The high $r^{2}$ value (0.999) supports the method. The binding parameters for sulfonamide + HSA interactions recovered from (1) were listed in Tables 1 and 2. The agreement between the calculated and experimental results (Figures 1 and 2 ) gives considerable support to the use of (1). $\delta_{A}^{\theta}$ and $\delta_{B}^{\theta}$ values for sulfonamide + HSA interactions are positive, indicating that in the low and high concentrations of the sulfonamide, the HSA structure is stabilized. These results suggest that the antioxidant property of HSA increased. $p=1$ indicates that the binding is noncooperative.

For a set of identical and independent binding sites, a plot of $\left(\Delta q / q_{\max }\right)$ [HSA] versus $(\Delta q / q)$ [sulfon] should be a linear plot by a slope of $1 / g$ and the vertical-intercept of 


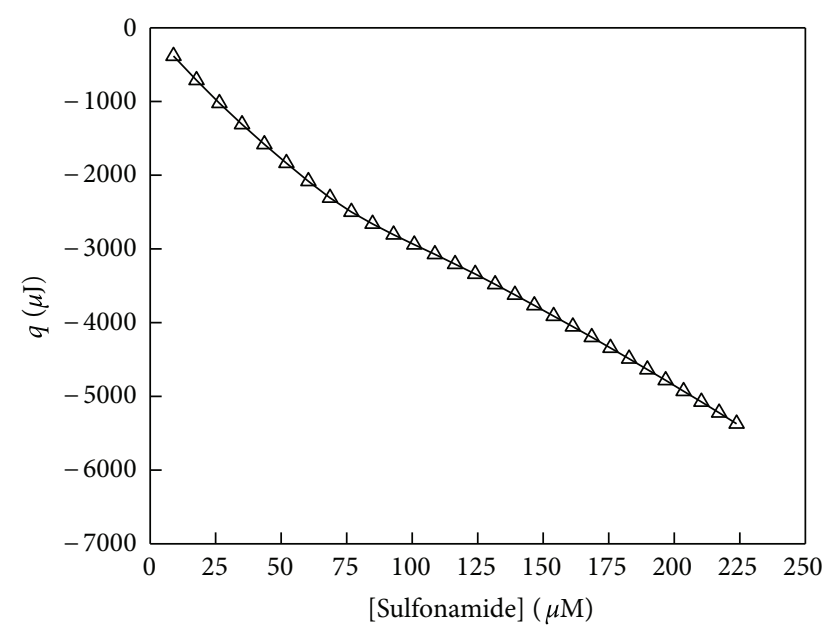

FIGURE 2: Comparison between the experimental heats $(\Delta)$ at $300 \mathrm{~K}$, for (sulfonamide + HSA) interactions and the calculated data (lines) via (1).

TABLE 1: Binding parameters for HAS + sulfonamide interaction. The interaction is both enthalpy and entropy driven, but the electrostatic interactions are more important than hydrophobic forces. $K_{a}$ values show that sulfonamide in the second class of binding sites has higher affinity for binding than the first class of binding sites. The positive values of $\delta_{A}^{\theta}$ and $\delta_{B}^{\theta}$ indicate that the antioxidant property of HSA increased as a result of its interaction with sulfonamide.

\begin{tabular}{lcc}
\hline Parameters & First binding sites & Second binding sites \\
\hline$p$ & 1 & 1 \\
$g$ & 1 & 4 \\
$K_{a} / \mathrm{L} \cdot \mathrm{mol}^{-1}$ & $2.2 \times 10^{6} \pm 250$ & $3.86 \times 10^{5} \pm 750$ \\
$\Delta H / \mathrm{kJ} \mathrm{mol}^{-1}$ & $-24.63 \pm 0.08$ & $-12.45 \pm 0.06$ \\
$\Delta G / \mathrm{kJ} \mathrm{mol}^{-1}$ & $-30.57 \pm 0.08$ & $-32.09 \pm 0.11$ \\
$\Delta S / \mathrm{kJ} \mathrm{mol}^{-1} \mathrm{~K}^{-1}$ & $0.02 \pm 0.003$ & $0.06 \pm 0.005$ \\
$\delta_{A}^{\theta}$ & 4.86 & \\
$\delta_{B}^{\theta}$ & & 4.76 \\
\hline
\end{tabular}

$K_{d} / g$, through which $g$ and $K_{d}$ can be obtained [15-19] as follows:

$$
\frac{\Delta q}{q_{\max }}[\mathrm{HSA}]=\left(\frac{\Delta q}{q}\right)[\text { sulfon }] \frac{1}{g}-\frac{K_{d}}{g},
$$

where $g$ is the number of binding sites, $K_{d}$ is the dissociation equilibrium constant, [HSA] and [sulfon] are the concentrations of HSA and sulfonamide or nanosulfonamide, respectively, $\Delta q=q_{\max }-q, q$ represents the heat value at a certain ligand concentration and $q_{\max }$ represents the heat value upon saturation of all HSA. If $q$ and $q_{\max }$ are calculated per mole of biomacromolecule, then the molar enthalpy of binding for each binding site $(\Delta H)$ will be $\Delta H=q_{\max } / g$. The best linear plots with the correlation coefficient value of 0.999 were obtained using amounts of -2670 and $-5400 \mu \mathrm{J}$ (equal to $-24.63,-49.81 \mathrm{~kJ} \mathrm{~mol}^{-1}$ ) for $q_{\max }$ in the first and second binding sites, respectively. Dividing the $q_{\max }$ amounts of $-24.63 \mathrm{~kJ} \mathrm{~mol}^{-1}$ by $g=1$, and $-49.81 \mathrm{~kJ} \mathrm{~mol}^{-1}$ by $g=4$,
TABLE 2: Binding parameters for HAS + nanosulfonamide interactions. The interaction is both enthalpy and entropy driven indicating that the electrostatic interactions are dominant. $K_{a}$ values show that nanosulfonamide has high affinity for binding to HSA. The positive value of $\delta_{A}^{\theta}$ indicates that the antioxidant property of HSA increased as a result of its interaction with nanosulfonamide. The negative $\delta_{B}^{\theta}$ value proves that nanosulfonamide dampened the anti-oxidant property of HSA in the high concentration of nanosulfonamide.

\begin{tabular}{lc}
\hline & Parameters \\
\hline$p$ & 1 \\
$g$ & 1 \\
$K_{a} / \mathrm{L} \cdot \mathrm{mol}^{-1}$ & $3.6 \times 10^{6} \pm 650$ \\
$\Delta H / \mathrm{kJ} \mathrm{mol}^{-1}$ & $-36.43 \pm 0.12$ \\
$\Delta G / \mathrm{kJ} \mathrm{mol}^{-1}$ & $-37.63 \pm 0.15$ \\
$\Delta S / \mathrm{kJ} \mathrm{mol}^{-1} \mathrm{~K}^{-1}$ & $0.004 \pm 0.001$ \\
$\delta_{A}^{\theta}$ & $2.65 \pm 0.06$ \\
$\delta_{B}^{\theta}$ & $-38.14 \pm 0.09$ \\
\hline
\end{tabular}

thus, gives $\Delta H=-24.63$ for the first binding sites and $\Delta H=$ $-12.45 \mathrm{~kJ} \mathrm{~mol}^{-1}$ for the second binding sites.

To compare all thermodynamic parameters in metal binding process for HSA, the change in standard Gibbs free energy $\left(\Delta G^{\circ}\right)$ should be calculated according to (6), whose value can be used in (7) for calculating the change in standard entropy $\left(\Delta S^{\circ}\right)$ of binding process:

$$
\begin{gathered}
\Delta G^{\circ}=-R T \ln K_{a} \\
\Delta G^{\circ}=\Delta H^{\circ}-T \Delta S^{\circ},
\end{gathered}
$$

where $K_{a}$ is the association binding constant (the inverse of the dissociation binding constant, $K_{d}$ ). The $K_{a}$ values are obtained as $22.1 \times 10^{5} \pm 250$ and $3.86 \times 10^{5} \pm 250 \mathrm{M}^{-1}$ for the first and second binding sites, respectively.

The results show that there are two sets of binding sites for sulfonamide. The interaction is both enthalpy and entropy driven, but the electrostatic interactions are more important than hydrophobic forces. It was found that there is 1 site in the first class of binding sites and 4 sites in the second class of binding sites. $K_{a}$ values show that sulfonamide in the second binding sites has higher affinity for binding than the first binding sites.

Energy of binding $\left(\Delta H=-36.43 \mathrm{~kJ} \mathrm{~mol}^{-1}\right)$ for nanosulfonamide with HSA is more negative than that of sulfonamide. Therefore, the energetic interaction between nanosulfonamide and HSA has become more favorable. The affinity of nanosulfonamide is roughly twice of sulfonamide, therefore reduces the drug dosage frequency, treatment time, and side effects. $K_{a}$ values show that nanosulfonamide has higher affinity for binding with HSA than sulfonamide. The more effectiveness of nanosulfonamide can be attributed to its small size which result in reducing drug toxicity, controlling time release of the drug and modification of drug pharmacokinetics and biological distribution. The positive $\delta_{A}^{\theta}$ value (Table 2) shows that nanosulfonamide (in around $30 \mu \mathrm{M}$ of nanosulfonamide) stabilizes HSA structure and increases the anti-oxidant property of HSA. The negative $\delta_{B}^{\theta}$ value indicates 
that nanosulfonamide dampened the anti-oxidant property of HSA in the high concentration domain (around $250 \mu \mathrm{M}$ of nanosulfonamide).

\section{Conflict of Interests}

There is no conflict of interest for any authors with Thermometric Digitam 3 software.

\section{Acknowledgment}

The financial support of Imam Khomeini International University is gratefully acknowledged.

\section{References}

[1] T. A. Waldmann, "Albumin cataboiism," in Albumin Structure, Function and Uses, V. M. Rosenoer, M. Oratz, and M. A. Rothschild, Eds., pp. 255-273, Pergamon, Oxford, UK, 1977.

[2] E. Bourdon and D. Blache, "The importance of proteins in defense against oxidation," Antioxidants and Redox Signaling, vol. 3, no. 2, pp. 293-311, 2001.

[3] T. J. Peters, All about Albumin, Academic Press, San Diego, Calif, USA, 1996.

[4] H. Watanabe, U. Kragh-Hansen, S. Tanase et al., "Conformational stability and warfarin-binding properties of human serum albumin studied by recombinant mutants," Biochemical Journal, vol. 357, no. 1, pp. 269-274, 2001.

[5] W. J. Long and J. W. Henderson, "Analysis of sulfa drugs on eclipse plus C18," 5989-5436EN, 2006.

[6] A. Göbel, A. Thomsen, C. S. McArdell et al., "Extraction and determination of sulfonamides, macrolides, and trimethoprim in sewage sludge," Journal of Chromatography A, vol. 1085, no. 2, pp. 179-189, 2005.

[7] S. A. Tilles, "Practical issues in the management of hypersensitivity reactions: sulfonamides," Southern Medical Journal, vol. 94, no. 8, pp. 817-824, 2001.

[8] W. E. Mueller and U. Wollert, "Circular dichroism studies on the interaction of four structurally related long acting sulfonamides with human and bovine serum albumin," Biochemical Pharmacology, vol. 25, no. 13, pp. 1459-1464, 1976.

[9] G. Rezaei Behbehani, A. A. Saboury, E. Poorakbar, and L. Barzegar, "Application of the extended solvation model for thermodynamic study of copper ion binding to Jack bean urease," Journal of Thermal Analysis and Calorimetry, vol. 102, no. 3, pp. 1141-1146, 2010.

[10] G. R. Behbehani, A. A. Saboury, and E. Yahaghi, "A thermodynamic study of nickel ion interaction with bovine carbonic anhydrase II molecule," Journal of Thermal Analysis and Calorimetry, vol. 100, no. 1, pp. 283-288, 2010.

[11] G. Rezaei Behbehani, A. A. Saboury, and F. Sabbaghy, "A calorimetric study on the interaction of zinc and cadmium ions with Jack bean urease," Chinese Journal of Chemistry, vol. 29, no. 3, pp. 446-450, 2011.

[12] G. Rezaei Behbehani, A. Divsalar, A. A. Saboury, F. Faridbod, and M. R. Ganjali, "A thermodynamic study on the binding of human serum albumin with lanthanum ion," Chinese Journal of Chemistry, vol. 28, no. 2, pp. 159-163, 2010.

[13] L. Barzegar, G. Rezaei Behbehani, and A. A. Saboury, "A thermodynamic study of zinc ion interaction with bovine carbonic anhydrase II at different temperatures," Journal of Solution Chemistry, vol. 40, no. 5, pp. 843-848, 2011.

[14] G. Rezaei Behbehani, A. A. Saboury, L. Barzegar, O. Zarean, J. Abedini, and M. Payehghdr, "A thermodynamic study on the interaction of nickel ion with myelin basic protein by isothermal titration calorimetry," Journal of Thermal Analysis and Calorimetry, vol. 101, no. 1, pp. 379-384, 2010.

[15] G. Rezaei Behbehani, A. A. Saboury, O. Zarean, L. Barzegar, and S. Ghamamy, "Thermodynamic study of myelin basic protein upon interaction with $\left[\mathrm{Hg}^{2+}\right]$ using extension solvation model," Chinese Journal of Chemistry, vol. 28, no. 5, pp. 713-718, 2010.

[16] A. A. Saboury, M. S. Atri, M. H. Sanati, and M. Sadeghi, "Application of a simple calorimetric data analysis on the binding study of calcium ions by human growth hormone," Journal of Thermal Analysis and Calorimetry, vol. 83, no. 1, pp. 175-179, 2006.

[17] G. Rezaei Behbehani, A. A. Saboury, S. Tahmasebi Sarvestani, M. Mohebbian, M. Payehghadr, and J. Abedini, "A thermodynamic study on the binding of theophylline with human serum albumin," Journal of Thermal Analysis and Calorimetry, vol. 102, no. 2, pp. 793-798, 2010.

[18] E. Tazikeh, G. Rezaei-Behbehani, A. A. Saboury et al., "Thermodynamic study of the binding of mercury ion to human growth hormone at different temperatures," Journal of Solution Chemistry, vol. 40, no. 4, pp. 575-586, 2011.

[19] A. A. Saboury, M. S. Atri, M. H. Sanati, A. A. MoosaviMovahedi, G. H. Hakimelahi, and M. Sadeghi, "A thermodynamic study on the interaction between magnesium ion and human growth hormone," Biopolymers, vol. 81, no. 2, pp. 120-126, 2006. 

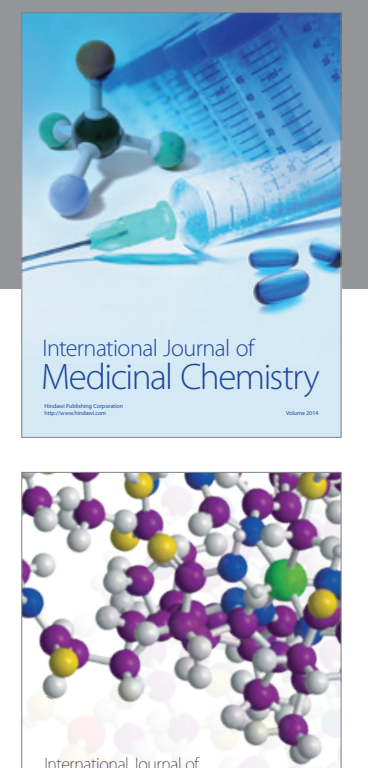

\section{Carbohydrate} Chemistry

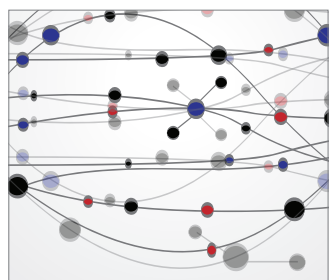

The Scientific World Journal
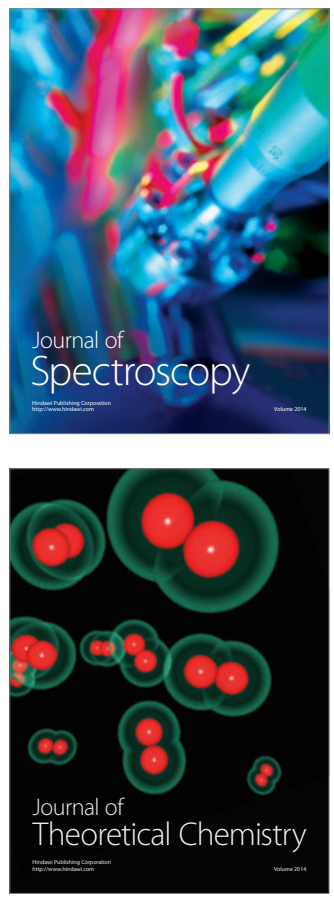
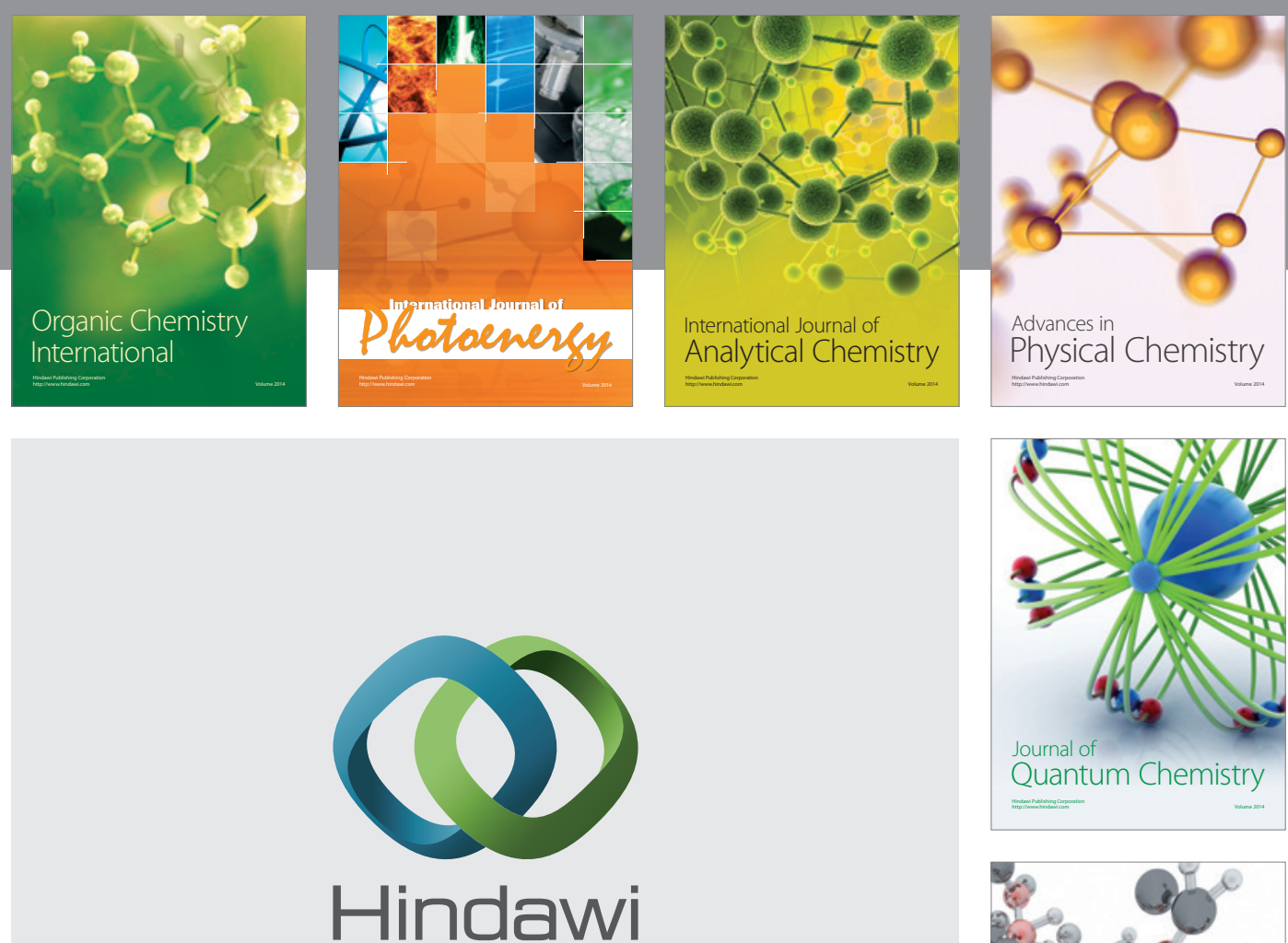

Submit your manuscripts at

http://www.hindawi.com

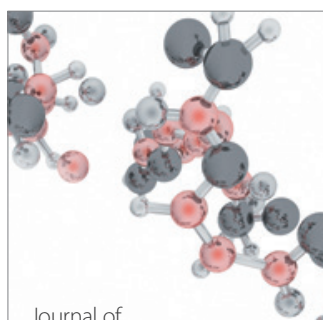

Analytical Methods

in Chemistry

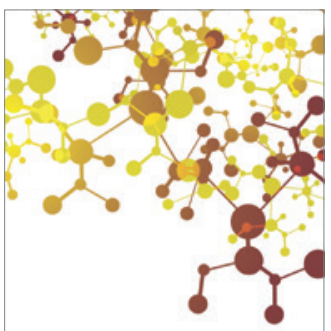

Journal of

Applied Chemistry

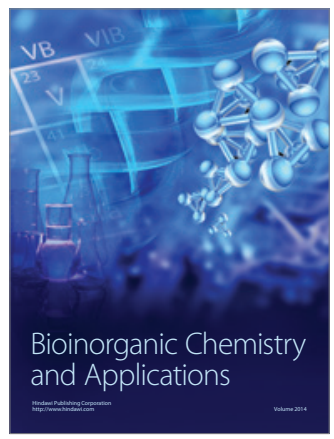

Inorganic Chemistry
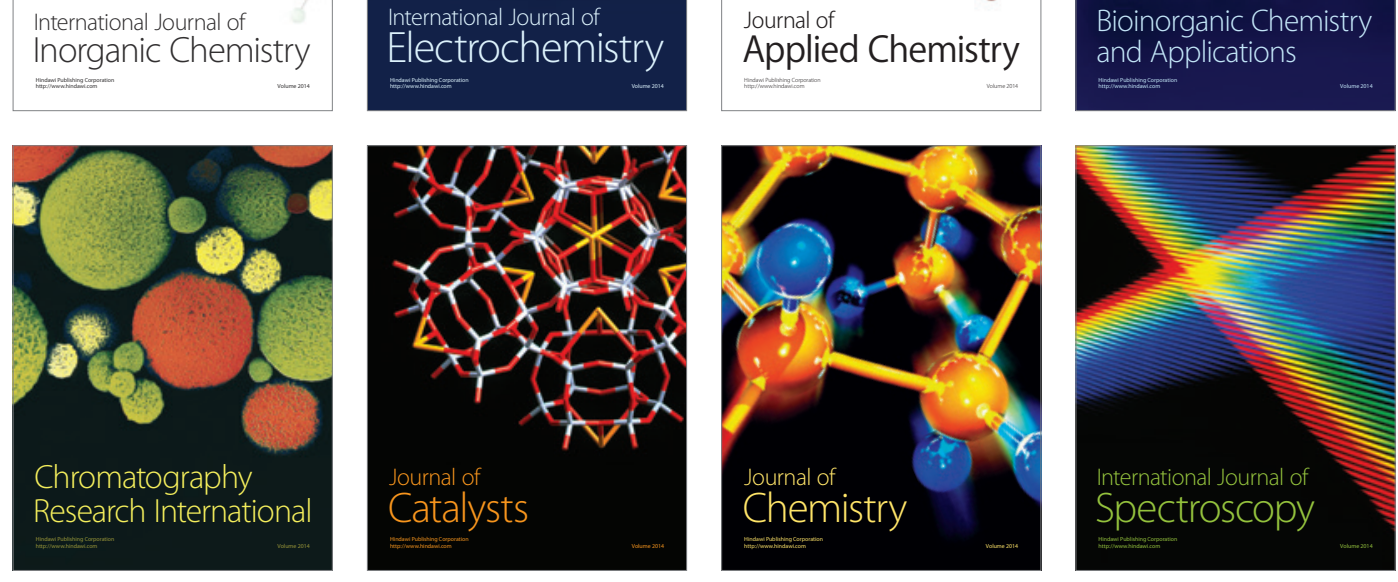